\title{
Modelling of Electric Vehicle with PID Controller Transfer Function using GA and Model-Reduced Order DRA Algorithm
}

\author{
Vasudeva Naidu Pudi ${ }^{1}$, GVSSN Srirama Sarma ${ }^{2}$, Srinivasa Rao Sura ${ }^{3}$, Prasad Bolla ${ }^{4}$ and N Siva \\ Mallikarjuna Rao ${ }^{5}$ \\ ${ }^{1,2}$ DoEEE, Matrusri Engineering College, India \\ ${ }^{3,4,5}$ DoEECE, GITAM, India \\ *Correspondence: Dr. Vasudeva Naidu Pudi; Email: pvdnaidu81 @ gmail.com
}

\begin{abstract}
In this paper, a model of an electric vehicle transfer function using GA and a model-reduced order discrete time realization (DRA) algorithm is presented. The electric vehicle (EV) control system regulates vehicle speed according to the driver's command signal and brings the vehicle to its equilibrium point, i.e., the desired speed under any abnormal conditions. The controller transfer function is designed based on EV's dynamic differential equations. An infinite-order transcendental transfer function for the EV model is approximated to find high-reliability discrete-time state-space reduced-order models (ROMs). Reduced the robustness and increased the predictions' accuracy of ROM output in terms of accurate numerical simulations of the EV transfer function. Also, the design of the PID controller parameter using optimization techniques (OT) for the tuning of conventional controllers for the desired speed is done using a linearized model. In this study, the performance of a conventional PID controller is compared with that of a GA-based PID controller. In terms of maximum overshoot, peak time, rising time, settling time, and steady state error, the simulation results show that the suggested controller is preferable.
\end{abstract}

Keywords: Electric Vehicle, Vehicle Control, PID Controller, Genetic Algorithm, Ho-Kalman method, Discrete Time Realization (DRA) Algorithm.

ARTICLE INFORMATION
Author(s): Vasudeva Naidu Pudi, GVSSN Srirama Sarma, Srinivasa Rao
Sura, Prasad Bolla N Siva Mallikarjuna Rao
Received: Dec 04, 2021; Accepted: Jan 31, 2022; Published: Feb 28, 2022;
e-ISSN: 2347-470X;
Paper Id: IJEER120105;
Citation: doi.org/10.37391/IJEER.090407
Webpage-link:
https://ijeer.forexjournal.co.in/archive/volume-9/ijeer-090407.html

\section{INTRODUCTION}

The world's auto industry has started to transform itself from a combustion-based industry to a new energy-based one. As a result, the development of electric vehicles accelerated. Due to their capability to recharge seamlessly, they are more competent than traditional vehicles [1]-[2]. Aside from buses and trains, electric vehicles are also used for off-road transportation like submarines and surface vessels. In the mid19th century, the advent of electricity led to the development of the EV. As a result, the vehicle became more practical and comfortable. Due to the advantages of electric vehicles, more people are considering buying electric cars instead of traditional ones. A BLDC motor is the main component of an electric vehicle. Before the advent of electric vehicles, internal combustion engines were considered the best choice for transportation. Presently, research is going on the design of adaptive electric vehicle (EV) control and its improved form. The control system of an EV has become a typical component of the cutting-edge vehicles for drivers' comfort in significant distance ventures and the security of the travelers by lessening the dangers of rapid mishaps. It is essentially speed control, which deals with the rules of battery and converter control as indicated by speed necessities [3]-[4].

The main methodology and objectives of this paper are as follows:

1. Modeling of the unit delay closed loop PID controller transfer function $\mathrm{G}(\mathrm{s})$ of the electric vehicle using the traction force equation, aerodynamic drag force expression, rolling resistance and gravity force expression [6]-[9], [11].

2. Conversion of EV transfer function $G(s)$ to the discrete-time form using a "realization algorithm "[16-20].

3. Design of longitudinal control of the EV after the construction of a reduced-order state-space model, to longitudinal control of the EV to improve the dynamic execution of the vehicle [5]. Generally, longitudinal control concepts are designed for automated automobiles and trucks operating on a cooperative highway with different disturbances [15]. The researchers designed a vehicle control system using different conventional controllers, viz., Proportional-Integral (PI), Proportional-Integral-Derivative (PID) for linear models. So, in this paper PID controller with Genetic Algorithm (GA) is designed for flexible tuning of the parameters [10]-[13] which is very difficult task in the conventional methodology. The objective of this study is to increase the speed of response by the way of reducing $T_{s}$ and maintain $M_{p}$ within the defined range [25]-[26]. In this paper, the designed simulation model of a closed loop vehicle controller with disturbances is validated through the simulation results. 


\section{THE VEHICLE DYNAMIC MODEL}

\subsection{Vehicle Dynamic Model}

Figure 1 depicts the vehicle's comprehensive longitudinal dynamic model. The propulsion system is described as a firstorder transfer function with time delay and force saturation, and the vehicle dynamics are nonlinear [6], [11], [14].

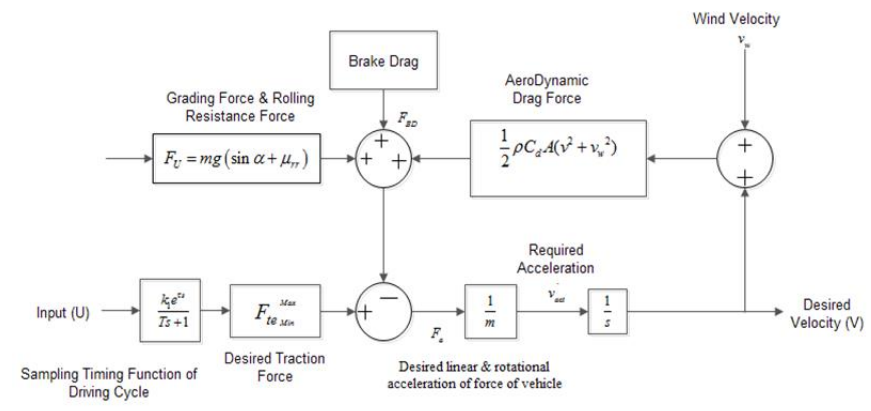

Figure 1. Vehicle longitudinal Dynamic model

Newton's Law is used to develop the fundamental dynamic formula for the vehicle model.

Total tractive Force

$$
F_{t}=\left(\frac{1}{2} \rho C_{d} A\left(v^{2}+v_{w}^{2}\right)+F_{r r}+F_{B D}+F_{h c}\right)+F_{a}
$$

Aero Dynamic Drag force $\left(\mathrm{F}_{\mathrm{adw}}\right)=\frac{1}{2} \rho C_{d} A\left(v^{2}+v_{w}{ }^{2}\right)$

Aerodynamic coefficient $\left(C_{d}\right)=0.3-0.5$ for sallon or hatchback vehicles \& the lowest value of $C_{d}$ is 0.04 (length to diameter of vehicle body is 2.4 ).

Rolling Resistance Force $\left(F_{r r}\right)=\mu_{r r} * m g$

$\mu_{r r}=\mu_{0}+\mu_{S}\left(\frac{V}{100}\right)^{2.5}$

The values $\mu_{0} \& \mu_{s}$ depend on inflation pressure of the tire. $\mu_{r r} \simeq$ (Area of deflection/wheel radius), the value of

$\mu_{r r}=0.015$ for a radial ply tyre \& 0.005 for a tyre of vehicle and gravity $(g)=9.81 \mathrm{~m} / \mathrm{s}$

( $\therefore$ Note that the rolling force is computed to be zero if the prior actual speed is ' 0 ')

Hill Climbing Force $F_{h c}=m^{*} g \sin \alpha$

Grade angle ( $\alpha$ ) is positive angle for an incline slope of road $\&$ negative angle for a decline slope of road.

Constant friction force from sticking brake pads:

Brake Drag $\left(\mathrm{F}_{\mathrm{BD}}\right)=$ constant road force input by user As shown in Figure 2, total tractive Force:

$F_{t}=\left(\frac{1}{2} \rho C_{d} A\left(v^{2}+v_{w}{ }^{2}\right)+F_{r r}+F_{B D}+F_{h c}\right)$
Starting point the vehicle motion equation is represented by equation

$m \frac{d v(t)}{d t}=F_{t}(t)-\left(\frac{1}{2} \rho C_{d} A\left(v(t)^{2}+v_{w}(t)^{2}\right)+F_{r r}(t)+F_{B D}(t)+F_{h c}(t)\right)$

$F_{h c}(t)=0$ (assumed no hill grading)

$F_{B D}(t)=0$ (assumed no brake drag during the movement of the vehicle)

$F_{r r}(t)=0$ (assumed no rolling resistance force during the movement of the vehicle)

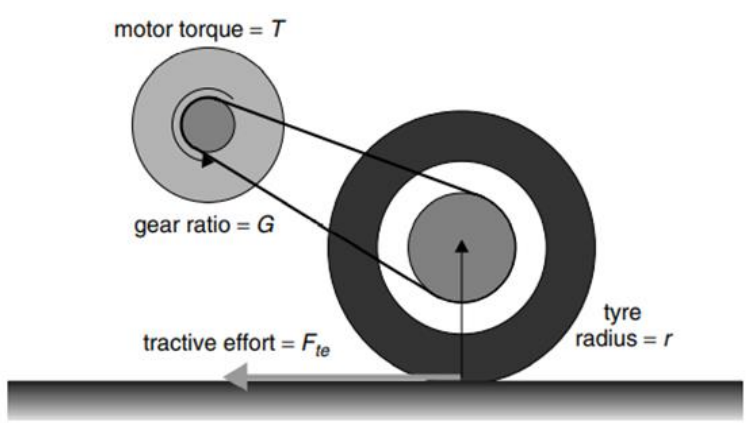

Figure 2. Tractive Force on Vehicle

Initial speed of vehicle is ' $v(0)$ ' wind speed ' $v_{w}$ ' (opposite to the vehicle speed), hence the vehicle speed of vehicle ( $\left.v(0)+v_{w}\right)$. To find the output equations by applying this zero initial condition in block diagram shown in figure.1.

$\dot{v}=\frac{1}{m}\left(F_{t}-\frac{1}{2} \rho C_{d} A\left(v(t)+v_{w}\right)^{2}\right)$

$\dot{F}_{t}=\frac{1}{T}\left(K_{1} u(t-T)-\left.F_{t}\right|_{\left(F_{t \min } \leq F_{t} \leq F_{t \min }\right)}\right)$

Differentiated the above equations (8) \& (9)

$\frac{d \dot{v}}{d t}=\frac{d\left(\frac{1}{m}\left(F_{t}-\frac{1}{2} \rho C_{d} A\left(v^{2}+v_{w}^{2}\right)\right)\right)}{d t}=\frac{1}{m}\left(\dot{F}_{t}-\rho C_{d} A\left(v+v_{w}\right)\right)$

$\frac{d \dot{F}_{t}}{d t}=\frac{1}{T}\left(K_{1} \Delta u(t-T)-\left.\dot{F}_{t}\right|_{\left(F_{t \min } \leq F_{t} \leq F_{t \min }\right)}\right)$

And the output equation becomes $y=\Delta v$, where $\Delta v$ is disturbance. Now desired velocity $v(t)=v(0)+\Delta v$ and the time delay of the engine $\Delta u(t-T)$. Solution of state equations provides the transfer function $\frac{\Delta V(s)}{\Delta U(s)}$.

The Laplace transformation expression of tractive force $F_{t}(s)$ is

$F_{t}(s)=\frac{K_{1} e^{-\tau s}}{\left(T_{1} s+1\right)}$ where $\left(F_{t \min } \leq F_{t} \leq F_{t \min }\right)$

' $K_{1}$ ' is the product of gains, $e^{-\tau s}$ is the delay factor

The Laplace transformation velocity expression of aerodynamic drag force is 


$$
\begin{aligned}
& F_{a d}(s)=\left(\frac{1}{m s+\left(2 \rho C_{d} A\left(v(0)+v_{w}\right)\right.}\right)=\left(\frac{\left(\frac{1}{\left.2 \rho C_{d}^{A\left(v(0)+v_{w}\right)}\right)}\right)}{\left(\frac{m}{\left.2 \rho C_{d}^{A\left(v(0)+v_{w}\right)}\right) s+1}\right)}\right. \\
& G_{\text {plant }}(s)=\frac{\Delta V(s)}{\Delta U(s)}=F_{t}(s) * F_{a d}(s)=\left(\frac{\left(\frac{1}{2 \rho C_{d} A\left(v(0)+v_{w}\right)}\right)}{\left(\frac{m}{2 \rho C_{d} A\left(v(0)+v_{w}\right)}\right) s+1}\right)\left(\frac{K_{1} e^{-\tau s}}{\left(T_{1} s+1\right)}\right)
\end{aligned}
$$

The power series expansion of $e^{-\tau s}=\frac{1}{1+\tau s}$ gives the time delay.

$$
G_{\text {plant }}(s)=\frac{\Delta V(s)}{\Delta U(s)}=\left(\frac{\left(\frac{1}{2 \rho C_{d} A\left(v(0)+v_{w}\right)}\right)}{\left(\frac{m}{2 \rho C_{d} A\left(v(0)+v_{w}\right)}\right) s+1}\right)\left(\frac{K_{1} e^{-\tau s}}{\left(T_{1} s+1\right)}\right)\left(\frac{1}{\tau s+1}\right)
$$

\section{METHODLOGY}

\subsection{Longitudinal Conventional Vehicle Models}

The longitudinal control problem has logical and control aspects. Based on the current conditions, the vehicle position in future time steps is dealt with the logical aspect for the system's best performance [5]. The desired position can be attained and maintained by controlling the vehicle based on control aspects for a comfortable and safe ride within certain constraints. Out of three models available under the logical and control aspects of longitudinal control problems, the point mass longitudinal model is used in this proposed work.

\subsubsection{The point-mass longitudinal model}

The point-mass longitudinal model is based on Newton's 2nd law of motion, as shown in the Figure 3. The drive force acting on the vehicle due to its propulsion system and the aerodynamic and frictional drag force, which is a non-linear function of the vehicle velocity, mentioned in section 2.1 results in the net force acting on an EV.

$$
\dot{v}=\frac{1}{m}(-\alpha V+u)
$$

Where $\mathrm{m}=$ mass of the vehicle, $\mathrm{V}=$ longitudinal speed of the vehicle, $\alpha=$ linearized drag co-efficient acting upon the vehicle assuming small deviations from the desired speed and $\mathrm{u}=$ drive force acting upon the vehicle through the control system as mentioned in Eq. (7).

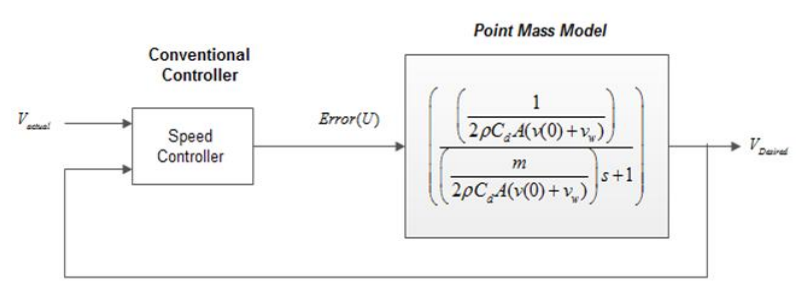

Figure 3. Point-mass Model

3.1.2 Proposed Methodology: Optimizing Techniques (OT) Non-Linear Longitudinal Electric Vehicle model

The main challenge of the EV regulator arrangement is maintaining the output speed of the system at the input signal level. The solution is to implement a controller function that dynamically augments or modifies the open loop function as

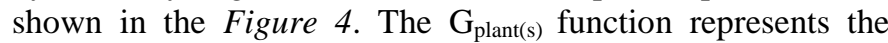
system, which includes the battery management system, DCDC converter, motor, and controller. The controller parameters of the EV are resolute primarily. Prior to taking any choice on controller configuration, design determinations have been made deliberately. In this design, two influencing parameters for better traditional response of the system, i.e., $T_{s}$ less than 5 $s$ and $M_{p}$ less than $10 \%$, are met. Generally, linear model controllers focus on PID controllers, GA-genetic algorithm, PSO-Practical Swarm optimization, etc.

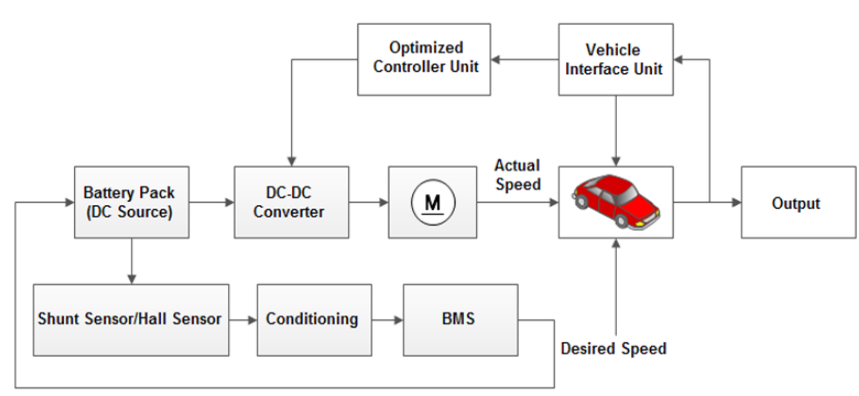

Figure 4. Block diagram of Closed Loop EV control System

4. MODEL -ORDER REDUCTION DISCRETE-TIME REALIZATION ALGORITHM (DRA) FOR GIVEN EV
TRANSFER FUCTION

Eq. (18) is converted into unit-pulse response using the discrete-time realization method (DRA). To create a reducedorder state-space model, Ho-Kalman algorithm approaches are used.

$G(s)=\left(\frac{\left(\frac{1}{2 \rho C_{d} A\left(v(0)+v_{w}\right)}\right)}{\left(\frac{m}{2 \rho C_{d} A\left(v(0)+v_{w}\right)}\right) s+1}\right)\left(\frac{K_{1} e^{-\tau s}}{\left(T_{1} s+1\right)}\right)\left(\frac{1}{\tau s+1}\right)\left(K_{P}+\frac{K_{i}}{s}+K_{d} s\right)$

Continuous-time transfer function with PID controller, $G(s)=G_{P I D}(s) \times G_{\text {plant }}(s)$ in the Laplace domain. From Figure 3.

$V(s)=\left(G_{P I D}(s) G_{\text {plant }}(s)\right) \times U(s), \quad$ state-space realization of system is given in eq. (19) [21]-[23].

$$
\left.\begin{array}{c}
x[k+1]=A x[k]+B u[k] \\
v[k]=C x[k]+D u[k]
\end{array}\right\}
$$

Step for the overall DRA process [26]-[27]: 
Step 1: Get the continuous-time impulse response $G(t)$ by applying the inverse discrete Fourier transform (IDFT).

Step 2: obtained to continue unit pulse response $\&$ discretetime unit-pulse response using $G_{\text {pulse }}(t)=G_{\text {step }}(t)-G_{\text {step }}\left(t-T_{S}\right) \& g_{k}=G_{\text {pulse }}\left(k T_{s}\right)$.

Step 3: The necessary \& sufficient condition for stable and proper system to apply DRA is that Hardy space $G \infty, g(k)$ as an element.

Step 4: Using the deterministic Ho-Kalman technique, generate a discrete-time state-space realization.

To derive the Ho-Kalman method, multiply the subsequent matrices together

Observability Matrix $\mathrm{O}=\left[\begin{array}{l}C \\ C A \\ C A^{2} \\ C A^{3} \\ \vdots \\ C A^{n-1}\end{array}\right]$, Controllability Matrix $C=$

$\left(\begin{array}{llll}B & A B & A^{2} B \cdots & A^{n-1} B\end{array}\right)$

$O C=\left[\begin{array}{ccccc}C B & C A B & C A^{2} B & \ldots & C A^{n-1} B \\ C A B & C A^{2} B & C A^{3} B & \ldots & \\ C A^{2} B & C A^{3} B & C A^{4} B & \cdots & \\ \vdots & & & \ddots & \\ C A^{n-1} B & & & \cdots & C A^{2 n-2} B\end{array}\right] ; \quad G=O C=\left[\begin{array}{ccccc}g_{1} & g_{2} & g_{3} & \ldots & g_{n} \\ g_{2} & g_{3} & g_{4} & \cdots & \\ g_{3} & g_{4} & g_{5} & \cdots & \\ \vdots & \vdots & & \ddots & \\ g_{n} & & & \cdots & g_{2 n+1}\end{array}\right]$

Where $\mathrm{n}$ is infinite, $G_{\infty}$ is the infinite Hankel matrix, D gives us $g_{o}$ directly.

Where $k>n$ and $l>n$, therefore $O_{k} \neq O$ and $C_{l} \neq C$ [27].

Finite Hankel Matrix $\left(G_{k, l}\right)$ is

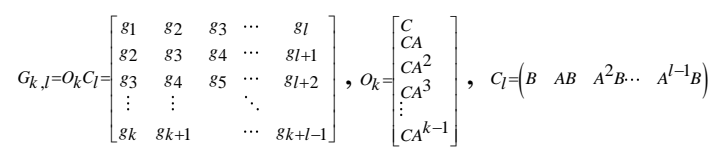

Finite Hankel matrix is mentioned in Eq. (22).

The single value dimension of the Hankel matrix $G_{k, l}$ is calculated as per the Eq. (23) and the system order ' $n$ ' is identify as the number of "large" singular values.

$$
\begin{aligned}
G_{k, l}= & u \sum V^{T}=u \sum^{1 / 2} \sum^{1 / 2} V^{T} \\
& =u \sum V^{T}=u \Sigma^{1 / 2} \Sigma^{1 / 2} V^{T} \\
& =u \Sigma^{1 / 2} T T^{-1} \Sigma^{1 / 2} V^{T} \\
& =\left(u \Sigma^{1 / 2} T\right)\left(T^{-1} \Sigma^{1 / 2} V^{T}\right)=O_{k} C_{l}
\end{aligned}
$$

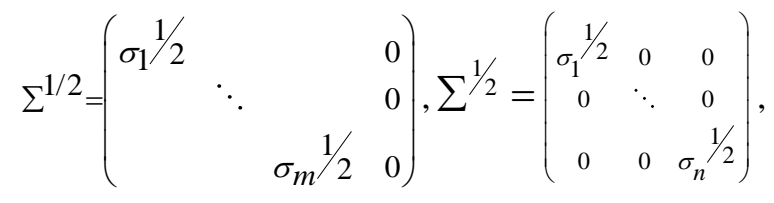

$\Sigma^{1 / 2}=\left(\begin{array}{ccc}\sigma_{1}^{1 / 2} & & \\ & \ddots & \\ & & \sigma_{n}^{1 / 2} \\ 0 & 0 & 0\end{array}\right)$ When $m<n, \quad m=n, \quad m>n$ respectively, $U=\left[u_{1}, \cdots, u_{m}\right] \in \sqcup^{m \times n}$, and $U^{T} U=I$, and $u_{i}$ and $v_{i}$ are the left $\&$ right or input singular vectors of $A$ and $\sum \in R^{m \times n}$ is a (zero-padded) diagonal matrix containing singular values $\sigma_{i}$ of the matrix $A$. The factoring of $G_{k, l}$ is factored into $O_{k}$ and $C_{l}$, depends on the choice of transformation matrix $T$. For $\mathrm{T}$ to be invertible it should be identity matrix on simplicity grounds.

The resultant matrix obtained by applying shift property of a Hankel matrix $G_{k, l}$ by one block row

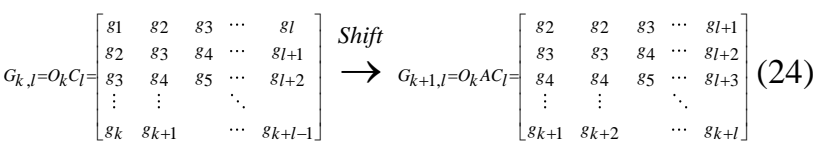

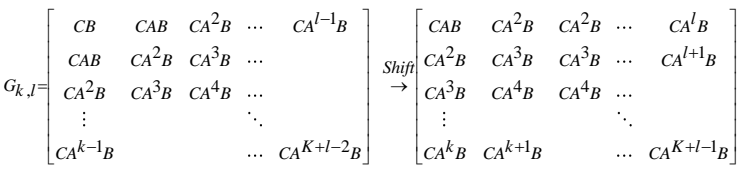

$\hat{A}=O_{k}^{\dagger} G_{k+1, l^{\dagger}} C_{l}^{\dagger}, \hat{B}=B$ and $\hat{C}=C$

Where $\hat{B}=B$ is the expanded controllability matrix's first block column \& $\hat{C}=C$ is the extended observability matrix $O_{k}$ first block row, we set our estimate $\hat{D} \square g_{0}\left(D=g_{0}=G[0]=\lim _{s \rightarrow \infty} G(s)\right.$.

Above all the steps of given transfer function $G(s)$ are implemented and results and discussions are explained in results \& discussion section.

\subsection{Optimization of objective function using Optimization Techniques (OT)}

The objective function of $E q$. (30) is minimized by choosing the optimal values of the controller's parameters $\mathrm{k}_{\mathrm{p}}, \mathrm{k}_{\mathrm{i}} \& \mathrm{k}_{\mathrm{d}}$ based on the genetic algorithm known as GA. The characteristics of the step response for the optimal values of $\mathrm{k}_{\mathrm{p}}, \mathrm{k}_{\mathrm{i}} \& \mathrm{k}_{\mathrm{d}}$ are given in Table 2 and step response is shown in Figure $9 b$.

The characteristic equation of the EV controller with unit delay is given by

$$
1+G_{\text {Plant }}(s) G_{P I D}(s)=0
$$




$$
1+\left(\frac{\left(\frac{1}{2 \rho C_{d} A\left(v(0)+v_{w}\right)}\right)}{\left(\frac{m}{2 \rho C_{d} A\left(v(0)+v_{w}\right)}\right) s+1}\right)\left(\frac{K_{1} e^{-\tau s}}{\left(T_{1} s+1\right)}\right)\left(\frac{1}{\tau s+1}\right)\left(K_{P}+\frac{K_{i}}{s}+K_{d} s\right)=0
$$

The Equation (30) represents objective function for computing the value of $K_{P}, K_{i}$ and $K_{d}$, for parameters mentioned in the Table 2.

Minimize $f\left(K_{p}, K_{i} \& K_{d}\right)=$

$$
1+\left(\frac{\left(\frac{1}{2 \rho C_{d} A\left(v(0)+v_{w}\right)}\right)}{\left(\frac{m}{2 \rho C_{d} A\left(v(0)+v_{w}\right)}\right) s+1}\right)\left(\frac{K_{1} e^{-\tau s}}{\left(T_{1} s+1\right)}\right)\left(\frac{1}{\tau s+1}\right)\left(K_{P}+\frac{K_{i}}{s}+K_{d} s\right)=0
$$

Subjected to: $\quad 0 \leq K_{P} \leq 5,0 \leq K_{i} \leq 1$

$$
0 \leq K_{d} \leq 5 \text { and error } \leq 0
$$

\subsubsection{Genetic Algorithm (GA)}

GA is used to optimize the parameters of a control system on a global scale. Using Ziegler and Nichols' approach and traditional methods, the optimization gets more complicated. Global optimization of a control system involves keeping a set of solutions called population. The solution selection step selects the parents of the population and then uses the resulting offspring for the next generation [24]-[25].

Table 1. The parameters of genetic algorithm

\begin{tabular}{|l|l|}
\hline Genetic Algorithm (GA) & Value \\
\hline Parameter & 3 \\
\hline No of dimensions & 50 \\
\hline No. of populations & 50 \\
\hline No, of iterations & 0.5 \\
\hline Mutation probability & 0.01 \\
\hline Crossover probability & $0-5$ \\
\hline$K_{p}$ Range & $0-1$ \\
\hline$K_{i}$ Range & $0-5$ \\
\hline$K_{d}$ Range & \\
\hline
\end{tabular}

\section{RESULTS AND DISCUSSION}

Table 2. The Vehicle parameters \& Values

\begin{tabular}{|l|l|}
\hline Parameter & Value \\
\hline Vehicle Mass in kg & $1500 \mathrm{~kg}$ \\
\hline Aerodynamic Drag Coefficient $\left(C_{a}=0.5 \rho C_{d} A\right)$ & Range: 1.19 to 2.0 \\
\hline Driving Coefficient $\left(K_{1}=\frac{m}{2 \rho C_{d} A\left(v(0)+v_{w}\right)}\right)$ & $\begin{array}{l}\text { Max: 3500 N.M, } \\
\text { Min: 3500 N.M }\end{array}$ \\
\hline Time Constant for Vehicle Propulsion & $1.0 \mathrm{~s}$ \\
\hline Steady State Vehicle Speed $(v(0))$ in $\mathrm{m} / \mathrm{s}$ & $25-60$ \\
\hline Wind Vehicle Speed $\left(v_{w}\right)$ in $\mathrm{m} / \mathrm{s}$ & 5 \\
\hline Delay Time $(\tau)$ & $0.2 \mathrm{~s}$ \\
\hline
\end{tabular}

\subsection{Results \& Discussion on Model Order Reduction Using DRA algorithm}

After the optimization characteristic equation (30), $\mathrm{K}_{\mathrm{p}}, \mathrm{K}_{\mathrm{i}}$, and $\mathrm{K}_{\mathrm{d}}$ values are obtained as $3.845,0.086$, and 3.484 respectively. After substituting the above values in $\mathrm{G}(\mathrm{s})$, the model order reduction (DRA) technique is used. The Model an Electric Vehicle with a PID Controller Transfer Function using GA and the Model-Reduced Order DRA Algorithm steps mentioned in section 4 are followed for the implementation of the DRA algorithm.

Step 1: The approximate continuous-time impulse response of $G(s)$ is computed using IDFT and the exact continuous-time impulse response of $G(s)$ are coincident, demonstrating that the DFT method provides a good approximation to the impulse response.

Step 2: To comparison between the DRA-approximated step response and the true step response of the system. Again, we see excellent agreement between the two signals in Figure $5 a$ $\& 5 b$.

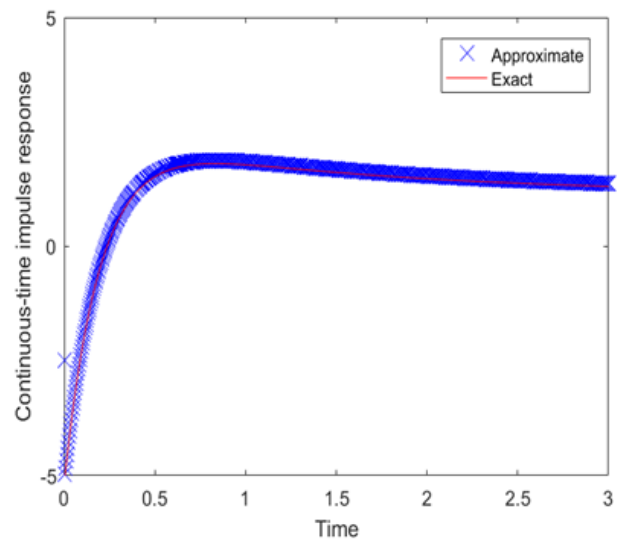

Figure 5a. Continuous-time step response of G(S)

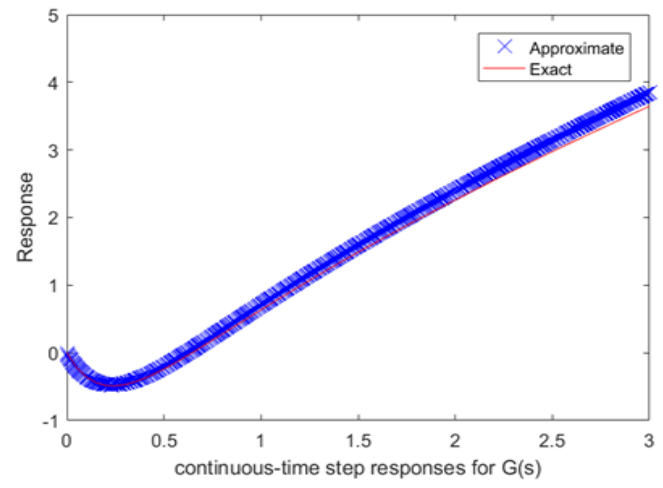

Figure 5b. Continuous-time step response of $\mathrm{G}(\mathrm{S})$

Step 3: To find approximate a continuous-time impulse response in discrete time, and its output must be scaled by the sampling period $T_{s}$ to achieve the true unit-pulse response and comparison between the unit-pulse responses produced by the DRA approximate method and MATLAB's exact method shown in Figure 6. 


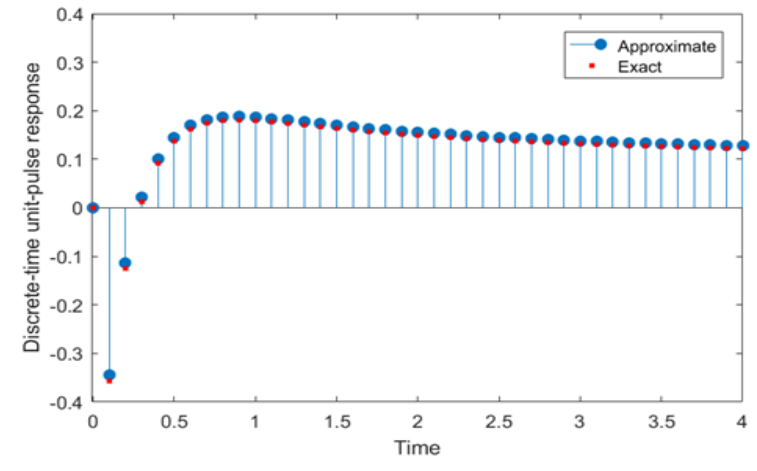

Figure 6a. Unit-pulse responses of $\mathrm{G}(\mathrm{S})$ produced by the DRA approximate method

Step 4: To determine estimated matrices $\hat{A}, \hat{B}$ and $\hat{C}$ using Ho-Kalman algorithm \& selects a reduced-order model dimension of $n=2^{20}[27]$.

$\hat{A}=\left[\begin{array}{rr}0.9982 & 0.0604 \\ -0.0604 & 0.5506\end{array}\right], \hat{B}=\left[\begin{array}{l}0.3475 \\ 0.6835\end{array}\right], \hat{C}=\left[\begin{array}{ll}0.3475 & -0.6835\end{array}\right]$

$\hat{D}=0=g_{0} \rightarrow\left(D=g_{0}=h[0]=\lim _{s \rightarrow 0} H^{*}(s)=0\right)$

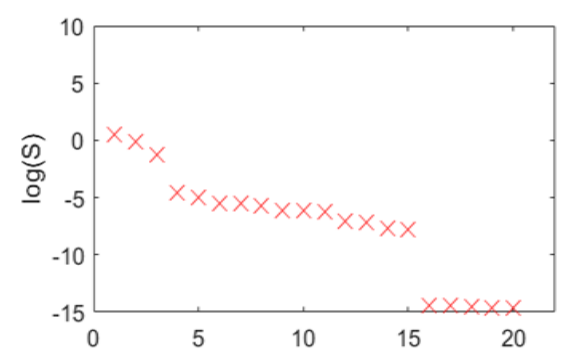

Figure 6b. MATLAB's exact method \& reduced-order model dimension

The Figure 7 shows that the final DRA model unit-pulse response agrees very well with the exact unit-pulse response. The implemented reduced order model system is absolutely stable \& proper system.



Figure 7: Final DRA model unit-pulse response of G(S)

\subsection{Results \& Discussion on Model Order Reduction Using GA algorithm}

The constraints of genetic algorithm (GA) are shown in Table 1. Initially in reduced EV controller system with conventional PID control has been designed in MATLAB/Simulink and the model is shown in Figure 8. The desired signal or driver command signal is unit step signal. This desired signal is compared with actual output signal from the plant and error is given to the PID controller. The discrete ROM is successfully implemented to identify the stability of proposed system which is one of the best upgradations in the work done by the predecessors $(9,10,11,12,13)$ in this area of research. The external force aerodynamic forces, rolling resistance forces etc., have been considered in the proposed vehicle model used in this paper which are not accounted the previous researchers ${ }^{(28)}$.

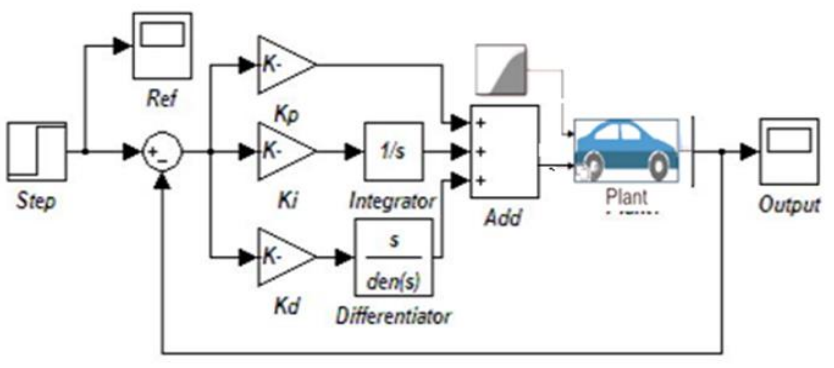

Figure 8. EV speed control with conventional PID controller

Figure 8 shows the step response of velocity of the closed loop EV control system with conventional PID controller after simulation. The characteristics of step response are shown in Table 3.

One of the important elements that must be addressed during controller design to assess the performance of controller designs for linear model vehicle control systems is deciding on a criterion that quantifies the quality of the response. Figure 9 shows the step response closed loop vehicle control system with GA based PID controller. The most common parameters for comparison and to know the stability of the system are the percentage overshoot $(\% \mathrm{OS})$, peak time $\left(T_{p}\right)$, settling time (Ts), rise time (Tr) and the percent steady state error (\%Error). Table 3 summarizes all the responses specification values. It is evident that genetic algorithm (GA) based PID controller is more stable controller from comparison with conventional controller characteristics.

The investigation using Root locus for analysis of transient \& stability behavior yields that EV system is stable and with good dynamic performance. Figure 10a shows the root locus curve for the system using the GA based PID controller. A bode plot shown in Figure $10 \mathrm{~b}$ is to assess information about system's frequency response. 


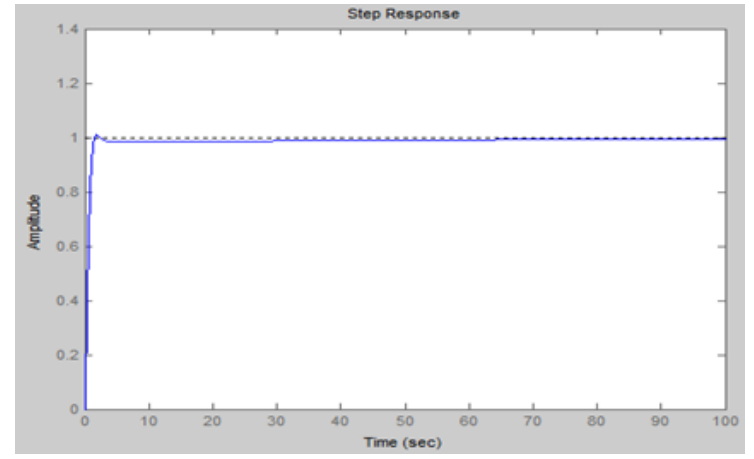

Figure 9. The step response of the closed loop EVcontrol system using GA based PID controller

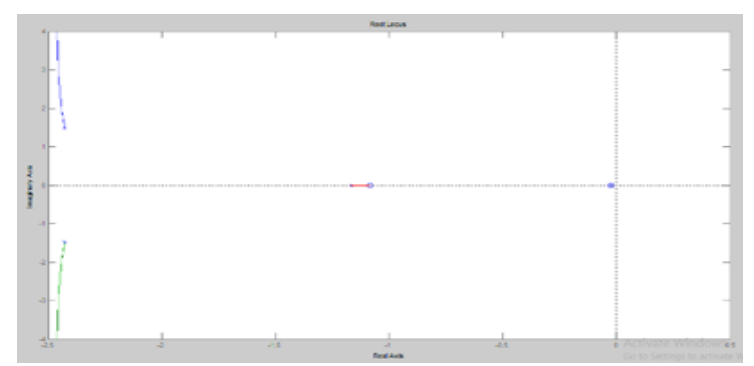

(a)

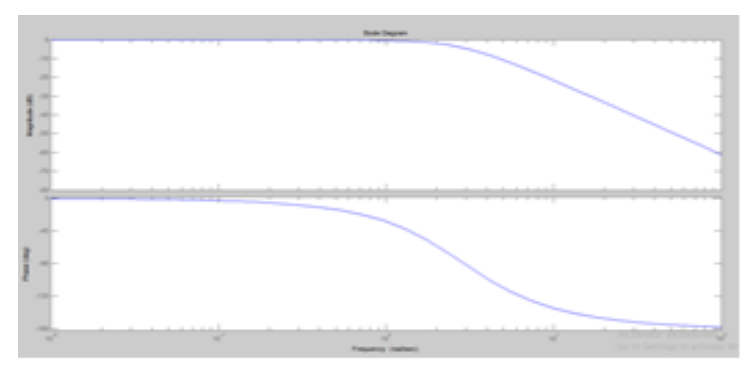

(b)

Figure 10 (a) \& (b). Time response \& Frequency Response analysis of the EV closed lopp control system using GA based PID controller

Table 3. Comparison Values Conventional PID Controller and PID controller tuning Using GA

\begin{tabular}{|l|l|l|}
\hline \multirow{2}{*}{ Parameters } & Vehicle control Using \\
\cline { 2 - 3 } & Conventional PID Controller & $\begin{array}{l}\text { PID Tunning with } \\
\text { GA }\end{array}$ \\
\hline $\mathrm{Kp}$ & 4.5 & 3.845 \\
\hline $\mathrm{Ki}$ & 0.1 & 0.086 \\
\hline $\mathrm{Kd}$ & 3.5 & 3.484 \\
\hline Rise Time (Tr) & 0.945 & 1.0088 \\
\hline Settling Time (Ts) & 1.46 & 1.3928 \\
\hline $\begin{array}{l}\text { Settling Min } \\
\left(\mathrm{T}_{\text {smin }}\right)\end{array}$ & 1.108 & 0.9729 \\
\hline $\begin{array}{l}\text { Settling Max } \\
\left(\mathrm{T}_{\text {smax }}\right)\end{array}$ & 1.210 & 1.0099 \\
\hline Overshoot & 1.147 & 0.9977 \\
\hline Undershoot & 0 & 0 \\
\hline Peak & 1.00 & 1.0099 \\
\hline Peak Time (Tp) & 2.15 & 1.7457 \\
\hline
\end{tabular}

\section{CONCLUSION}

In this paper, a GA-based PID controller for the EV with model reduced order by the DRA algorithm through a relevant mathematical model is discussed. The robustness is reduced and accuracy of the predictions of the reduced-order model output with respect to precise numerical simulations of the EV transfer function is increased by applying DRA algorithm. The proposed vehicle model has taken into account external factors such as aerodynamic forces and rolling resistance forces. Under any disturbance the vehicle can be brought back to desired speed with the help of sensors that are based on the tuning of GA based PID controller. The result analysis shows that the proposed controller outperforms the other controllers in terms of maximum overshoot, peak time, rise time, settling time, and steady state error. The output rejects disturbance and high-frequency noise, and operates in the most stable mode, according to the system's frequency response.

\section{ACKNOWLEDGMENTS}

Our thanks to the experts who have contributed towards development of the template.

\section{REFERENCES}

[1]. Muller, R. Nocker, G. Daimler-Benz AG and Stuttgart. 1992. "Intelligent Cruise Control with Fuzzy Logic", IEEE Intelligent Vehicles '92 Symposium. DOI:10.1109/IVS.1992.252252.

[2]. St. Germann and R. Isermann "Nonlinear Distance and Cruise Control for Passenger Cars, American Control Conference", IEEE American Control Conference Jun 1995. DOI: 10.1109/ACC.1995.532083.

[3]. Lewis, Paul H. and Yang Chang "Basic Control System Engineering". Prentice-Hall, Inc. New Jersey, USA 1997. ISBN:978-0-13-597436-0.

[4]. Khaled Sailan and Klaus.Dieter Kuhnert "Modeling and Design of Cruise Control System with Feed forward for All Terrian Vehicles" ICAITA, SAI, SEAS, CDKP, CMCA-2013 pp. 339-349, 2013. DOI:10.5121/csit.2013.3828.

[5]. Frank, Andrew A., S. J. Liu, S. C. Liang. "Longitudinal Control Concepts for Automated Automobiles and Trucks Operating on a Cooperative Highway". Society of Automotive Engineers Technical Paper Series (891708) 1989. https://doi.org/10.4271/891708

[6]. Thomas E.Marlin. 2002. Process Control: "Designing Processes and Control Systems for Dynamic Performance". 2nd Ed. Mc. Graw Hill. ISBN: 978-0070393622.

[7]. Pan Zhao, Jiajia Chen, Yan Song, Xiang Tao, Tiejuan Xu and Tao Mei, "Design of a Control System for an Autonomous Vehicle Based on Adaptive-PID” Int J Adv Robotic Sy, 2012, Vol. 9, 44:2012. DOI: $10.5772 / 51314$

[8]. Ogata, Katsuhiko. 2002. "Modern Control Engineering". 4th Ed. Prentice-Hall, Inc. New Jersey, USA. ISBN: 978-0136156734

[9]. Kim, D.; Moon, S.; Park, J.; Kim, H.J.; Yi, K. "Design of an Adaptive Cruise Control / Collision Avoidance with Lane Change Support for Vehicle Autonomous Driving", ICROS-SICE International Joint Conference, Fukuoka International Congress Center, Japan, August 1821, 2009. INSPEC Accession Number: 10982336

[10]. Sahoo, A.K.; Panda, G.; Pradhan, P.M. "Efficient Design of Pulse Compression Codes Using Multi objective Genetic Algorithm", World Congress on Nature \& Biologically Inspired Computing (NaBIC 2009), 2009. DOI: $10.1109 / \mathrm{NABIC} .2009 .5393731$

[11]. Khairuddin Osman, Mohd. Fuaad Rahmat, Mohd Ashraf Ahmad. "Modelling and Controller Design for a Cruise Control System". 5th International Colloquium on Signal Processing \& Its Applications (CSPA), 2009. DOI: 10.1109/CSPA.2009.5069228

[12]. Vedam, N.; Diaz-Rodriguez, I.; Bhattacharyya, S.P. "A novel approach to the design of controllers in an automotive cruise-control system". Industrial Electronics Society, IECON 2014 - 40th Annual Conference of the IEEE, Pages: 2927 - 2932, Year: 2014. DOI: 10.1109/IECON.2014.7048925

[13]. M. K. Rout, D. Sain, S. K. Swain, S. K. Mishra. "PID controller design for cruise control system using genetic algorithm", 2016 International 
Conference on Electrical, Electronics, and Optimization Techniques (ICEEOT), 2016. DOI: 10.1109/ICEEOT.2016.7755502

[14]. Vehicle modeling equations are from T. Gillespie, "Fundamentals of Vehicle Dynamics," Society of Automotive Engineers Inc, 1992. ISBN of 978-1-56091-199-9

[15]. Moler, C., and Van Loan, C., "Nineteen Dubious Ways to Compute the Exponential of a Matrix, Twenty-Five Years Later," SIAM Review, 45(1), 2003, pp. 3-49. DOI:10.1137/S00361445024180

[16]. D. DePalma, Efficient Computation of Reduced-Order Models of Lithium-Ion Cells, Master's thesis, University 868 of Colorado Colorado Springs, 2014

[17]. A. V. Oppenheim, R. W. Schafer, Discrete-Time Signal Processing, Prentice Hall Press, Upper Saddle River, NJ, 861 USA, 3rd edition, 2010. ISBN: 978-0131988422

[18]. Smith, K., Electrochemical Modeling, Estimation and Control of Lithium-Ion Batteries, Ph.D. dissertation, The Pennsylvania State University, 2006.

[19]. Ho, B.L., and Kalman, R.E., "Effective Construction of Linear State Variable Models from Input/Output Functions," Regelungstechnik, 14(12), 1966, pp. 545-8. https://doi.org/10.1524/auto.1966.14.112.545

[20]. Jacobsen, T., and West, K., "Diffusion Impedance in Planar, Cylindrical and Spherical Symmetry," Electrochimica Acta, 40(2), 1995, pp. 25562. DOI:10.1016/0013-4686(94)E0192-3

[21]. Lee, J.L., Chemistruck, A., and Plett, G.L., "Discrete-Time Realization of Transcendental Impedance Functions, with Application to Modeling Spherical Solid Diffusion,” Journal of Power Sources, 206, 2012, pp. 367-377. DOI:10.1016/j.jpowsour.2012.01.134

[22]. Franklin, G.F., Powell, J.D., and Workman, M., "Digital Control of Dynamic Systems", 3rded., Addison-Wesley, 1998, pp. 187-210. ISBN13: 978-0-9791226-1-3

[23]. Juang, J.N., and Pappa, R.S., "Eigen system Realization Algorithm for Modal Parameter Identification and Model Reduction," Journal of Guidance, Control, and Dynamics, 8(5), 1985, pp. 620-27. https://doi.org/10.2514/3.20031

[24]. Naidu, PV, and B Basavaraja. "PSO-based optimization of PI regulator and VA loading of a SRF-based multiconverter DVR", International Journal of Engineering Science and Technology, 2014. DOI: 10.4314/ijest.v7i1.5

[25]. Vasudeva Naidu Pudi, Basavaraja Banakara. "PSO based optimal PI tunning of AHCC D-STATCOM", 2015 16th International Scientific Conference on Electric Power Engineering (EPE), 2015. 10.1109/EPE.2015.7161063

[26]. Albert Rodríguez, Gregory L. Plett, M. Scott Trimboli. "Comparing four model-order reduction techniques, applied to lithium-ion battery-cell internal electrochemical transfer functions", eTransportation, 2019. DOI:10.1016/J.ETRAN.2019.100009

[27]. Albert Rodríguez, Gregory L. Plett. "Controls-oriented models of lithium-ion cells having blend electrodes. Part 2: Physics-based reducedorder models", Journal of Energy Storage, 2017. DOI:10.1016/j.est.2017.02.006

[28]. D Kirti Swarup Jenaland Arockia Vijay Joseph "Dynamic Modelling and Control Design for a Vehicle in its Longitudinal Motion" Indian Journal of Science and Technology, Vol 9(30), August 2016. DOI: 10.17485/ijst/2016/v9i30/99019.

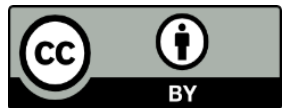

(C) 2021 by the Vasudeva Naidu Pudi, GVSSN Srirama Sarma, Srinivasa Rao Sura, Prasad Bolla and N Siva Mallikarjuna Rao. Submitted for possible open access publication under the terms and conditions of the Creative Commons Attribution (CC BY) license (http://creativecommons.org/licenses/by/4.0/). 